\title{
Prostate Segmentation with Local Binary Patterns Guided Active Appearance Models
}

\author{
Soumya Ghose ${ }^{a b}$, Arnau Oliver ${ }^{a}$, Robert Martí ${ }^{a}$, Xavier Lladó ${ }^{a}$, Jordi Freixenet ${ }^{a}$, Joan C. \\ Vilanova $^{c}$ and Fabrice Meriaudeau ${ }^{b}$ \\ ${ }^{a}$ Universitat de Girona, Computer Vision and Robotics Group, Campus Montilivi, Edifici-P \\ IV, 17071, Girona, Catalonia, Spain. \\ ${ }^{b}$ Université de Bourgogne, Le2i-UMR CNRS 5158, 12 Rue de la Fonderie, 71200, Le Creusot, \\ Bourgogne, France. \\ ${ }^{c}$ Clnica Girona, Carrer Joan Maragall 26, 17002, Girona, Catalonia, Spain.
}

\begin{abstract}
Real-time fusion of Magnetic Resonance (MR) and Trans Rectal Ultra Sound (TRUS) images aid in the localization of malignant tissues in TRUS guided prostate biopsy. Registration performed on segmented contours of the prostate reduces computational complexity and improves the multimodal registration accuracy. However, accurate and computationally efficient segmentation of the prostate in TRUS images could be challenging in the presence of heterogeneous intensity distribution inside the prostate gland, and other imaging artifacts like speckle noise, shadow regions and low Signal to Noise Ratio (SNR). In this work, we propose to enhance the texture features of the prostate region using Local Binary Patterns (LBP) for the propagation of a shape and appearance based statistical model to segment the prostate in a multi-resolution framework. A parametric model of the propagating contour is derived from Principal Component Analysis (PCA) of the prior shape and texture information of the prostate from the training data. The estimated parameters are then modified with the prior knowledge of the optimization space to achieve an optimal segmentation. The proposed method achieves a mean Dice Similarity Coefficient (DSC) value of $0.94 \pm 0.01$ and a mean segmentation time of $0.68 \pm 0.02$ seconds when validated with 70 TRUS images of 7 datasets in a leave-one-patient-out validation framework. Our method performs computationally efficient and accurate prostate segmentation in the presence of intensity heterogeneities and imaging artifacts.
\end{abstract}

Keywords: Prostate Segmentation, Active Appearance Model, Texture, Local Binary Patterns

\section{INTRODUCTION}

More than 670,000 people are diagnosed with prostate cancer every year worldwide ${ }^{1}$ accounting for over 32,000 deaths in Noth America. ${ }^{2}$ In clinical practice, TRUS guided needle biopsy is performed to confirm the presence of cancerous tissues in prostate. However, localization of malignant tissues in TRUS is difficult due to the low soft tissue contrast in TRUS images. Multi-core prostate biopsy is performed from 6 to 12 cores to improve positive detection of malignant tissue. ${ }^{3}$ MR images provide higher contrast for soft tissues of the prostate that allows a better localization of cancerous tissues. However, interventional MRI guided biopsy is expensive and complicated. The localization of malignant tissues could be improved by real-time registration of a 2D TRUS video sequence

Further author information: (Send correspondence to Soumya Ghose or Dr. Arnau Oliver or Prof. Fabrice Meriaudeau.)

Soumya Ghose: E-mail: soumyaghose@gmail.com,

Arnau Oliver: E-mail: aoliver@eia.udg.edu, Telephone: +34 972418878

Xavier Lladó: E-mail: llado@eia.udg.edu,

Robert Martí: E-mail: marly@eia.udg.edu,

Jordi Freixenet: E-mail: jordif@eia.udg.edu,

Joan C. Vilanova: E-mail: kvilanova@comg.cat,

Fabrice Meriaudeau: E-mail: fabrice.meriaudeau@u-bourgogne.fr, Telephone: +33(0)385731077

Medical Imaging 2011: Image Processing, edited by Benoit M. Dawant, David R. Haynor,

Proc. of SPIE Vol. 7962, 796218 - @ 2011 SPIE - CCC code: 0277-786X/11/\$18 · doi: 10.1117/12.877955

Proc. of SPIE Vol. 7962 796218-1 


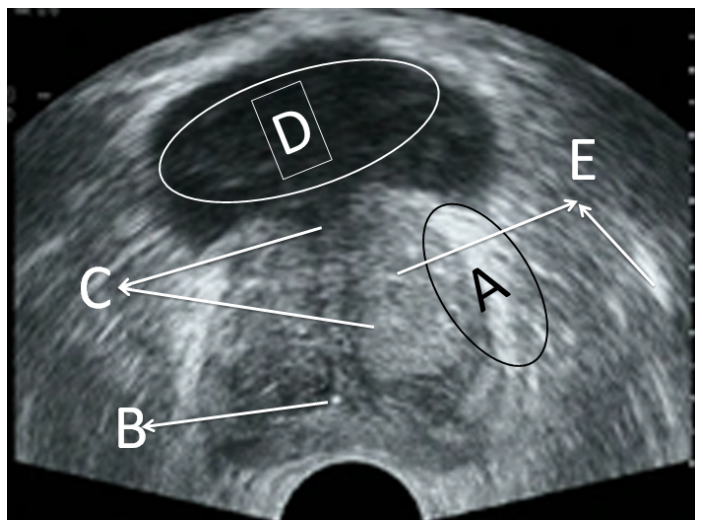

Figure 1. Prostate segmentation challenges , $\mathrm{A}=$ Low SNR, $\mathrm{B}=$ Micro Calcification, $\mathrm{C}=$ Intensity heterogeneity inside prostate, $\mathrm{D}=$ Shadow Artifacts, $\mathrm{E}=$ Speckle Noise.

with a 3D pre acquired MRI during TRUS guided biopsy. ${ }^{4}$ Registration performed on segmented contour of TRUS and MR images will aid in designing computationally efficient and accurate registration procedures. ${ }^{5}$ Thus, 2D segmentation of the prostate from TRUS video sequence is necessary for the process. However, automatic or semi-automatic accurate segmentation of the prostate in TRUS image is a challenging task due to low SNR, intensity heterogeneities inside the prostate gland and presence of imaging artifacts like the speckle noise and shadow regions. Low SNR produces spurious and broken edges with traditional edge detection filters and intensity heterogeneities inside the prostate gland inhibit the design of a global descriptor for the prostate. Added to these shadow artifacts, speckle noise and micro-calcifications is a hindrance to accurate segmentation for the segmentation algorithms. The challenges involved in accurate prostate segmentation in TRUS images are illustrated in Fig. 1.

To address the challenges of prostate segmentation in TRUS images Shen et al. ${ }^{6}$ and Betrouni et al. ${ }^{7}$ proposed to use prior prostate shape information in their models. Prior shape information made the models robust to imaging artifacts and detected true prostate edges in low SNR. Yan et al. ${ }^{5}$ proposed to incorporate prior shape information of the prostate in a partial active shape model framework. The model selectively discarded salient points for building the shape model to improve segmentation accuracy in presence of shadow artifacts in TRUS images. Gong et al. ${ }^{8}$ proposed to use deformable super ellipse to segment the prostate. Shape constraints of the model was effective in achieving impressive segmentation results. It has been demonstrated by Zhan et al. ${ }^{9}$ that incorporating shape and intensity prior information in prostate segmentation methods improve the segmentation accuracy. Cootes et al. ${ }^{10}$ provided an efficient framework for combining shape and intensity prior in their Active Appearance Model (AAM). AAM framework was used by Medina et al. ${ }^{11}$ to segment prostate in 2D TRUS images with an overlap ratio of $96 \%$. However, time complexity involved with AAM is high making the model unsuitable for real-time procedures. ${ }^{12}$ Larsen et al. ${ }^{13}$ proposed to use truncated wavelet coefficients in AAM framework to improve on segmentation accuracy of facial images and achieve reduction in segmentation time.

In this paper, we propose a novel AAM that is propagated by texture features of Local Binary Patterns $(\mathrm{LBP})^{14}$ transformed images in a multi-resolution framework. The motivation behind such an approach is to provide precise and concise texture information for the propagation of the AAM to improve on computational time and segmentation accuracy. The performance of our method is validated with 70 TRUS images of 7 datasets in a leave-one-patient-out validation framework. Experimental results show that our method is unaffected by low SNR, intensity heterogeneities and shadow and speckle noise. The key contributions of this work are:

- Enhancement of texture information of the prostate region from local binary patterns. ${ }^{14}$

- Use of the texture information in training and propagation of an active appearance model that improves on computational time and segmentation accuracy when compared to the traditional active appearance model. ${ }^{10}$ 


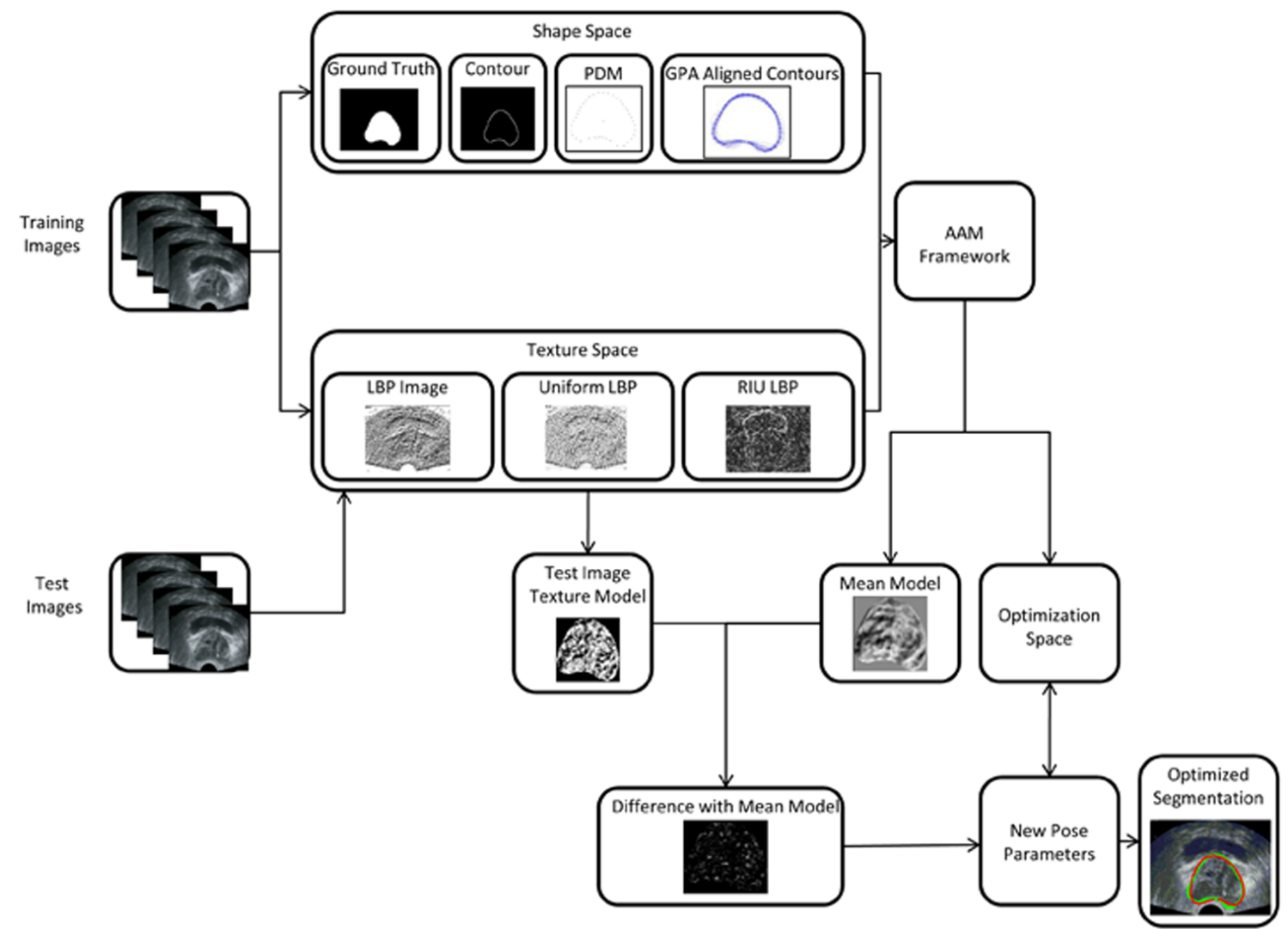

Figure 2. Schematic representation of our approach. The final segmentation is given in red contour and ground truth in green. Abbreviations used AAM = Active Appearance Model, PDM = Point Distribution Model, GPA = Generalized Procrustes Analysis, LBP = Local Binary Patterns, RIU = Rotational Invariant Uniform.

To the best of our knowledge this is the first attempt to use enhanced texture features of the prostate from LBP in training and propagation of AAM. The rest of the paper is organized as follows. The texture driven AAM is formulated in Section 2 followed by quantitative and qualitative evaluation of our method in Section 3. We finally draw conclusion in Section 4. The working schema of the model is illustrated in Fig. 2

\section{METHOD}

In our method the traditional AAM is adapted for the incorporation of texture information extracted from the LBP transformed image. Traditional AAM is presented here, followed by a comprehensive discussion about enhancement of texture information using LBP.

\subsection{Active Appearance Model}

Generalized Procrustes Analysis of the Point Distribution Model (PDM) ${ }^{15}$ built from manually segmented contours aligns the PDM in a common reference frame. Principal Component Analysis (PCA) of the aligned PDMs is used to identify the principal modes of shape variations. Similarly, PCA of the intensity distribution warped into correspondence using a piece wise affine warp and sampled from shape free reference is used to identify the principal modes of intensity variations. Principle modes of shape and intensity variations are combined and a third PCA ensures that primary components of combined model variations are identified. On completion of building the combined model, an unseen test image is segmented exploiting the prior knowledge of the nature of the optimization space. ${ }^{13}$ 
The model could be formalized in the following manner. In eq. 1 and 2 let $E\{s\}$ and $E\{t\}$ represent the shape and intensity models where $s$ and $t$ are the shape and the intensities of the corresponding training images, $\bar{s}$ and $\bar{t}$ denote the mean shape and intensity respectively, then $\Phi_{s}$ and $\Phi_{t}$ contain the first $p$ eigenvectors of the estimated joint dispersion matrix of shape and intensity and $\theta$ represents the corresponding eigenvalues.

$$
\begin{array}{r}
E\{s\}=\bar{s}+\Phi_{s} \theta \\
E\{t\}=\bar{t}+\Phi_{t} \theta
\end{array}
$$

In addition to the parameters $\theta$, four parameters, two translations, rotation and scale are represented by $\psi$. In order to infer the parameters of $\theta$ and $\psi$ of a previously unseen image, a Gaussian error model between model and pixel intensities is assumed. ${ }^{13}$ Furthermore, a linear relationship between changes in parameters and difference between model and image pixel intensities $\Delta t$ is assumed as shown in eq.3.

$$
\Delta t=X\left[\begin{array}{c}
\Delta \psi \\
\Delta \theta
\end{array}\right]
$$

$X$ is estimated from weighted averaging over perturbation of model parameters and training examples. Eq.3 is solved in a least square manner fitting error as shown in eq.4.

$$
\left[\begin{array}{c}
\widehat{\psi} \\
\widehat{\theta}
\end{array}\right]=\left(X^{T} X\right)^{-1} X^{T} \delta t
$$

The problem is computationally expensive. To reduce computational burden we propose to use a truncated basis for the representation of the pixel intensities achieved with LBP. However, this will introduce the additional time requirement of transformation of the image into a new representation. Since the transformation is based on sparse matrix, the computational burden can be considerably reduced as stated by Larsen et al. ${ }^{13}$

\subsection{Local Binary Patterns}

Ojala et al. ${ }^{14}$ proposed LBPs as computationally efficient discriminative transformations for texture analysis. Given a pixel in the image, LBP is computed by thresholding the neighborhood of the pixel. LBP of a pixel is defined as,

$$
L B P_{P, R}=\sum_{p=0}^{P-1} s\left(g_{p}-g_{c}\right) 2^{P}, \quad s(x)=\left\{\begin{array}{l}
1, x \geq 0 \\
0, x<0
\end{array}\right\}
$$

where, $g_{c}$ is the gray value of the centered pixel, $g_{p}$ are the gray values of the neighborhood pixels, $P$ is the number of neighbors and $R$ is the radius of the neighborhood. Signed difference $g_{p}-g_{c}$ is unaffected by change in mean luminance that provides gray scale invariance to the operator. Rotational invariance (RI) is defined as,

$$
L B P_{P, R}^{r i}=\min \left\{\operatorname{ROR}\left(L B P_{P, R, i}\right) \mid i=0,1 \ldots ., P-1\right\} \mid
$$

where $R O R(x, i\}$ performs a circular bit-wise right shift on the $P$-bit number $x, i$ times to assign a unique identifier to each rotation invariant local binary pattern. Ojala et. al. ${ }^{14}$ observed some fundamental patterns depicting the texture properties of an image under study. They named these patterns as uniform circular patterns of LBP transformed image. Uniform circular pattern is defined as in eq. 7 and 8

$$
L B P_{P, R}^{r i u 2}=\left\{\begin{array}{c}
\sum_{p=0}^{P-1} s\left(g_{p}-g_{c}\right) \text { if } U\left(L B P_{P, R}\right) \leq 2 \\
P+1 \text { otherwise }
\end{array}\right\}
$$




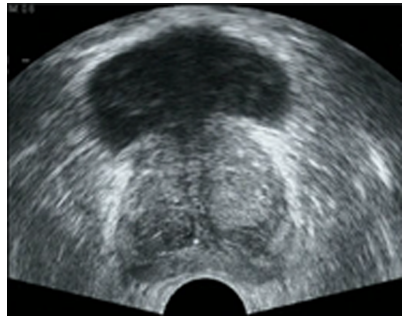

(a)

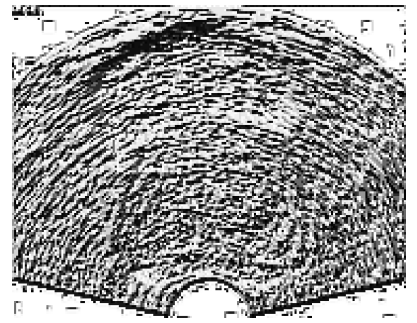

(b)

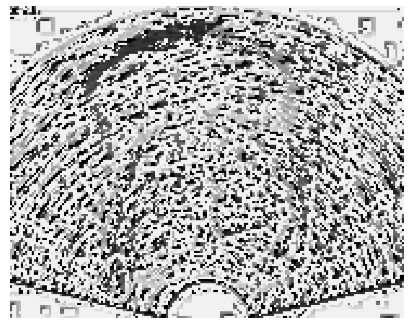

(c)

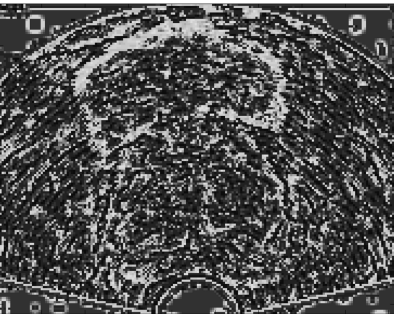

(d)

Figure 3. Variants of LBP transformed image using radius $=2$ and sample=16 (a) 2D TRUS image of the prostate, (b) LBP transformed image, (c) Uniform LBP transformed image, (d) Rotational invariant uniform LBP transformed image.

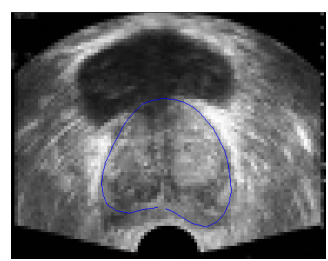

(a)

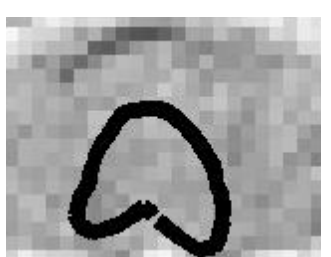

(b)

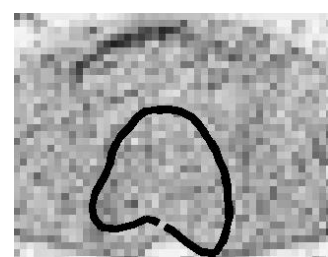

(c)

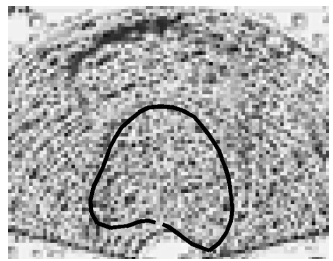

(d)

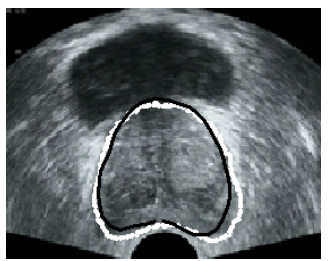

(e)

Figure 4. Multi-resolution functioning of the model (b) Manual initialization of the mean model by clicking on the center of the prostate, (b) Level 4 segmentation result, (c) Level 3 segmentation result, (d) Level 2 segmentation result, (e) Final segmentation result. Manual segmentation shown with white contour and the black contour show the segmentation achieved.

where

$$
\left(L B P_{P, R}^{U}\right)=\left|s\left(g_{p-1}-g_{c}\right)-s\left(g_{0}-g_{c}\right)\right|+\sum_{p=1}^{P-1}\left|s\left(g_{p}-g_{c}\right)-s\left(g_{p-1}-g_{c}\right)\right|
$$

Superscript riu2 refers to rotational invariant uniform patterns. Examples of LBP transformed images are given in Fig. 3

We have used LBP, uniform LBP and rotational invariant uniform LBP to produce LBP transformed representation of the image and used the PCA of the texture of the prostate region to propagate our AAM in a multi-resolution framework. The LBP transformed representation of the image is a truncated representation of the original intensity and hence fitting of the new image after LBP transformation is computationally efficient. Multi-resolution fitting improves segmentation accuracy and reduces the computational time of the process. The multi-resolution fitting of the model is illustrated in Fig. 4

\section{RESULTS}

We have validated the accuracy and robustness of our approach with 70 TRUS images of prostate in leave-onepatient-out evaluation strategy. The images are of resolution $538 \times 418$ obtained from 7 prostate datasets. Our method was implemented in Matlab 7 on an Intel Quad Core Q9550 processor of 2.83 Ghz processor speed and 8 GB RAM. We have used most of the popular prostate segmentation evaluation metrics like Dice Similarity Coefficient (DSC), 95\% Hausdorff Distance (HD), Mean Absolute Distance (MAD), Maximum Distance (MaxD), specificity, sensitivity, accuracy and mean segmentation time to evaluate our method. We have used two different values for the number of sample points $(P=16,8)$ and three different radii $(R=1,2,8)$ for the LBP and presented the best results in Table 1. $L B P_{16,2}$ corresponds to the traditional LBP as given in eq. $5, L B P_{16,2}^{U}$ represents the uniform LBP as given in eq. 8 , and $L B P_{16,8}^{r i u}$ represents rotational invariant uniform LBP as given in eq. 7. Subscript values denote neighbors and radius respectively. The results are given in Table 1

In Table 1 we observe that; in general LBP and different variants of LBP guided AAMs perform better than the traditional AAM comparing the distance metrics HD and MAD. This could be attributed to the fact that gray 
Table 1. Quantitative Comparison of AAMs

\begin{tabular}{|l|l|l|l|l|l|l|l|l|}
\hline Method & $\mathrm{DSC}$ & $\mathrm{HD}(\mathrm{mm})$ & $\mathrm{MAD}(\mathrm{mm})$ & $\mathrm{MaxD}(\mathrm{mm})$ & Specificity & Sensitivity & Accuracy & Time(sec) \\
\hline AAM & $0.93 \pm 0.03$ & $6.92 \pm 2.96$ & $2.15 \pm 0.94$ & $6.8 \pm 2.48$ & $0.89 \pm 0.03$ & $0.993 \pm 0.006$ & $0.97 \pm 0.009$ & $0.87 \pm 0.02$ \\
\hline$L B P_{16,2}$ & $0.94 \pm 0.01$ & $6.01 \pm 2.14$ & $1.66 \pm 0.46$ & $5.87 \pm 2.23$ & $0.91 \pm 0.02$ & $0.995 \pm 0.004$ & $0.98 \pm 0.005$ & $0.68 \pm 0.02$ \\
\hline$\left(L B P_{16,2}^{U}\right)$ & $0.95 \pm 0.01$ & $5.09 \pm 1.90$ & $1.50 \pm 0.41$ & $5.07 \pm 1.93$ & $0.91 \pm 0.01$ & $0.997 \pm 0.003$ & $0.98 \pm 0.004$ & $1.2 \pm 0.02$ \\
\hline$L B P_{16,8}^{r i u}$ & $0.94 \pm 0.02$ & $6.15 \pm 2.38$ & $1.78 \pm 0.75$ & $6.01 \pm 2.53$ & $0.90 \pm 0.03$ & $0.995 \pm 0.005$ & $0.97 \pm 0.01$ & $1.19 \pm 0.02$ \\
\hline
\end{tabular}

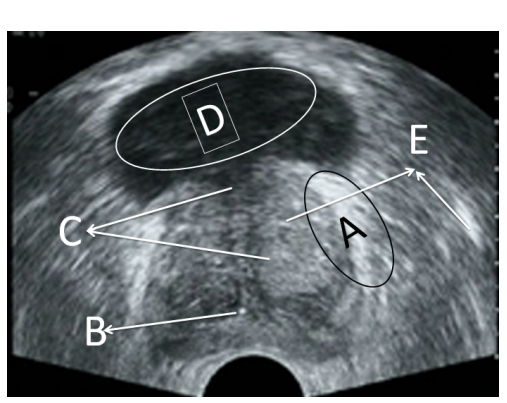

(a)

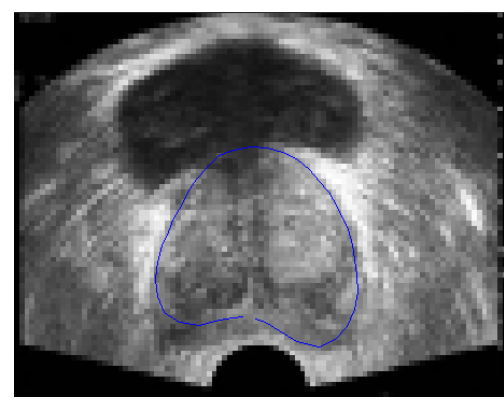

(b)

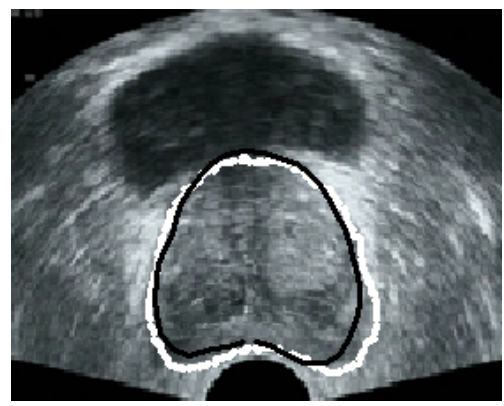

(c)

Figure 5. (a)Artifacts in TRUS image of the prostate, A=Low SNR, B=Micro Calcification, C=Intensity heterogeneity inside prostate, $\mathrm{D}=$ Shadow Artifacts, $\mathrm{E}=$ Speckle Noise. (b) Manual initialization of the mean model, (c) Final segmentation result.

scale invariance of the LBP operator helps gray-scale normalization of AAM, suppressing intensity heterogeneities, speckle noise and micro-calcifications in the process. LBP enhance underlying texture information of the prostate region. PCA of the LBP enhanced texture information reduces noise and helps in identifying principle modes of texture variation inside the prostate region. Surprisingly, computational complexities involved with traditional LBP method is less compared to traditional AAM considering the fact that the image had to be transformed into LBP map before fitting. On closer inspection it was revealed that average time for transforming 70 images into LBP map was 0.025 seconds and it produced an accurate representation of the prostate texture thereby reducing differences with the mean model. This in turn reduces the time for computationally expensive procedure of fitting a new image. The performance of uniform $\operatorname{LBP}\left(L B P_{16,2}^{U}\right)$ is better compared to the other variants of LBP. This is due to the fact that uniform LBP produces a uniform profile of the prostate region that is different from the neighboring tissue textures and therefore facilitates in improving the accuracy of segmentation. However, the procedure is computationally expensive compared to other variants of LBP. It is also observed that the performance of rotational invariant uniform $\operatorname{LBP}\left(L B P_{16,8}^{\text {riu }}\right)$ is almost comparable to traditional AAM.

The robustness of the proposed method against low SNR, intensity heterogeneities, shadow artifacts, speckle noise and micro-calcification is illustrated in Fig. 5. On initialization, a section of the mean model is located in a region of shadow artifact. The model successfully avoids the artifact and segments the prostate (black contour) with an accuracy of $97 \%$.

The mean model is initialized by clicking on the center of the prostate judged from visual inspection. However, the model could be initialized automatically with the assumption that the prostate is located at the center of the TRUS image. A more sophisticated approach would be to use an initial classification to initialize the mean model. Low standard deviation value associated with sensitivity, specificity and accuracy indicate that the final segmentation result is not affected by the manual initialization. To further validate our claim ten images were selected randomly and the mean model was initialized for 10 times in \pm 5 pixels around the center for uniform $\operatorname{LBP}\left(L B P_{16,2}^{U}\right)$. Mean accuracy of these initializations is $96.778 \pm 0.006 \%$. Low standard deviation associated with accuracy metric indicates that the process is indifferent to manual initialization as long as the initialization is close to the prostate center. To provide qualitative result of our method we present a subset of results obtained using uniform LBP operator $\left(L B P_{16,2}^{U}\right)$ in Fig. 6 

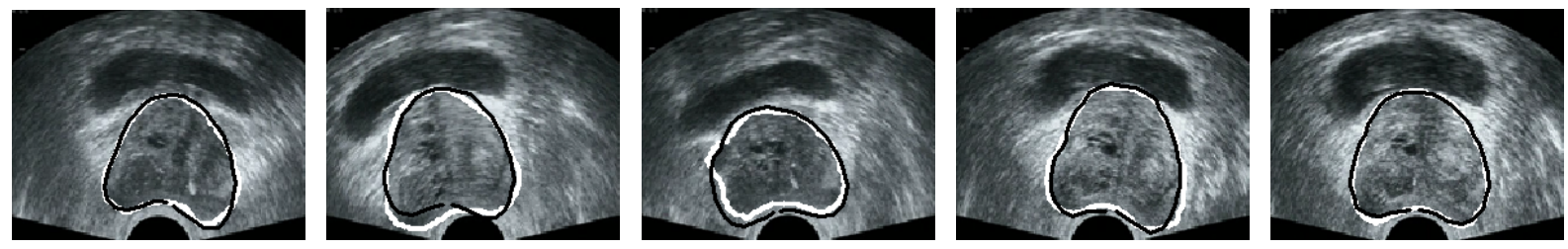

Figure 6. Qualitative segmentation results. The white contour gives the ground truth and the black contour gives the obtained result.

Table 2. Prostate Segmentation Evaluation: A Qualitative Comparison with the state-of-the-art algorithms for TRUS Images

\begin{tabular}{|l|l|l|l|l|}
\hline Reference & Year & Area Overlap Accuracy & Contour Accuracy & Datasets \\
\hline Yan $^{5}$ & 2009 & - & Average MAD 1.79 \pm 0.95 mm & 10 datasets \\
Abolmaesumi $^{16}$ & 2004 & Average Area overlap 98\% & - & 6 images \\
Gong $^{8}$ & 2004 & - & Mean error $1.36 \pm 0.6 \mathrm{~mm}$ & $125 \mathrm{images}$ \\
Betrouni $^{7}$ & 2004 & Average Area overlap $93 \pm 0.9 \%$ & Average distance $3.77 \pm 1.3$ pixels & 10 images \\
Shen $^{6}$ & 2003 & Average Area overlap error $3.98 \pm 0.97 \%$ & Average distance $3.2 \pm 0.87$ pixels & 8 images \\
Ladak $^{17}$ & 2000 & Average Area accuracy 90.1 $\pm 3.2 \%$ & Average MAD $4.4 \pm 1.8$ pixels & 117 images \\
\hline Our & & Average DSC 0.95 \pm 0.01 & Average MAD $1.50 \pm 0.41 \mathrm{~mm}$ & 7 datasets $/ 70$ \\
Method & & & & images \\
\hline
\end{tabular}

Comparison of different prostate segmentation methodologies is difficult in absence of public datasets and standardized evaluation metrics. In addition, the methods are developed with a wide variety of algorithms with specific application requirements. Nevertheless, to have an overall qualitative estimate of the functioning of our method we have compared our uniform LBP AAM with some of the state-of-the-art works in the literature (see Table 2) for TRUS images. Please note '-' in Table 2 means information is not available.

The average DSC value of our method with $0.95 \pm 0.01$ is comparable to mean DSC values achieved by Betrouni et al. ${ }^{7}$ and Ladak et al. ${ }^{17}$ and inferior to the area overlap measure of Abolmaesumi et al. ${ }^{16}$ of $98 \%$. However, it is to be noted that we have used more images (24 images) compared to 6 images of Abolmaesumi et al. Our MAD value of $1.50 \pm 0.41 \mathrm{~mm}$ is comparable to Gong et al. ${ }^{8}$ average distance value of $1.36 \pm 0.6 \mathrm{~mm}$ and to Yan et al. ${ }^{5}$ MAD of $1.79 \pm 0.95 \mathrm{~mm}$. From these observations we can safely conclude that qualitatively our method performs well in overlap and contour accuracy measures over some of the works in literature.

\section{CONCLUSION AND FUTURE WORKS}

A novel approach of AAM propagation using LBP enhanced texture profile with the goal of segmenting the prostate in 2D TRUS images has been proposed. Our approach is accurate, computationally efficient and robust to low SNR, intensity heterogeneities of the prostate tissues, shadow artifacts, speckle noise and microcalcification in TRUS images. While the proposed method is validated with prostate mid gland images, effectiveness of the method against base and apical slices is yet to be validated. Computational time of the process is fast but not suitable for real time segmentation of the prostate. We would like to explore the possibilities of optimization of our code and use CUDA platform to achieve necessary parallelization and hardware acceleration to achieve real-time segmentation of the prostate. We would like to explore the possibilities of automatic model initialization and extend the model for 3D segmentation of the prostate.

\section{ACKNOWLEDGMENTS}

This research has been funded by VALTEC 08-1-0039 of Generalitat de Catalunya, Spain and Conseil Régional de Bourgogne, France. 


\section{REFERENCES}

[1] "Prostate Cancer Statistics - Key Facts." http://info.cancerresearchuk.org/cancerstats/types/prostate, accessed [10th December 2010] (2009).

[2] "Prostate Cancer Statistics - Key Facts." www.cancer.gov/cancertopics/types/prostate, accessed [10th December 2010] (2010).

[3] Rodriguez, D. C., "Multi-core prostatic biopsy," Archivos españoles de urologia 55, 907-914 (2002).

[4] Xu, S., Kruecker, J., Turkbey, B., Glossop, N., Singh, A. K., Choyke, P., Pinto, P., and Wood, B. J., "Realtime MRI-TRUS fusion for guidance of targeted prostate biopsies," Computer Aided Surgery 13, 255-264 (2010).

[5] Yan, P., Xu, S., Turkbey, B., and Kruecker, J., "Optimal Search Guided by Partial Active Shape Model for Prostate Segmentation in TRUS Images," Proceedings of the SPIE Medical Imaging : Visualization, Image-Guided Procedures, and Modeling 7261, 72611G-72611G-11 (2009).

[6] Shen, D., Zhan, Y., and Davatzikos, C., "Segmentation of Prostate Boundaries from Ultrasound Images Using Statistical Shape Model," IEEE Transactions on Medical Imaging 22, 539-551 (2003).

[7] Betrouni, N., Vermandel, M., Pasquier, D., Maouche, S., and Rousseau, J., "Segmentation of Abdominal Ultrasound Images of the Prostate Using A priori Information and an Adapted Noise Filter," Computerized Medical Imaging and Graphics 29, 43-51 (2005).

[8] Gong, L., Pathak, S. D., Haynor, D. R., Cho, P. S., and Kim, Y., "Parametric Shape Modeling Using Deformable Superellipses for Prostate Segmentation," IEEE Transactions on Medical Imaging 23, 340-349 (2004).

[9] Zhan, Y. and Shen, D., "Deformable Segmentation of 3D Ultrasound Prostate Images Using Statistical Texture Matching Method," IEEE Transactions on Medical Imaging 25, 256-272 (2006).

[10] Cootes, T., Edwards, G., and Taylor, C., "Active Appearance Models," Book Series Lecture Notes in Computer Science Springer 1407, 484-498 (1998).

[11] Medina., R., Bravo, A., Windyga, P., Toro, J., Yan, P., and Onik, G., "A 2D Active Appearance Model For Prostate Segmentation in Ultrasound Images," 27th Annual International Conference of the IEEE Engineering in Medicine and Biology Society, 3363-3366 (2005).

[12] Wolstenholme, C. B. H. and Taylor, C. J., "Wavelet Compression of Active Appearance Models," Book Series Lecture Notes in Computer Science Springer 1679, 544-554 (1999).

[13] Larsen, R., Stegmann, M. B., Darkner, S., Forchhammer, S., Cootes, T. F., and Ersbll, B. K., "Texture Enhanced Appearance Models," Computer Vision and Image Understanding 106, 20-30 (2007).

[14] Ojala, T., Pietikäinen, M., and Mäenpää, T., "Multiresolution gray-scale and rotation invariant texture classification with local binary patterns," IEEE Transactions on Pattern Analysis and Machine Intelligence 24, 971-987 (2002).

[15] Cootes, T. F., Hill, A., Taylor, C. J., and Haslam, J., "The Use of Active Shape Model for Locating Structures in Medical Images," Image and Vision Computing 12, 355-366 (1994).

[16] Abolmaesumi, P. and Sirouspour, M., "Segmentation of Prostate Contours from Ultrasound Images," IEEE International Conference on Acoustics, Speech, and Signal Processing 3, 517-520 (2004).

[17] Ladak, H. M., Mao, F., Wang, Y., Downey, D. B., Steinman, D. A., and Fenster, A., "Prostate Segmentation from 2D Ultrasound Images," Proceedings of the 22nd Annual International Conference of the IEEE Engineering in Medicine and Biology Society 4, 3188-3191 (2000). 